\title{
Clinical and microbiological factors associated with early patient mortality from methicillin- resistant Staphylococcus aureus bacteremia
}

\author{
Tark Kim ${ }^{1}$, Yong Pil Chong ${ }^{2,3}$, Ki-Ho Park ${ }^{4}$, Kyung Mi Bang ${ }^{2,3}$, Su-Jin Park ${ }^{2,3}$, Sung-Han Kim², \\ Jin-Yong Jeong ${ }^{3,5}$, Sang-Oh Lee ${ }^{2}$, Sang-Ho $\mathrm{Choi}^{2}$, Jun Hee $\mathrm{Woo}^{2}$, and Yang Soo Kim²,3
}

\begin{abstract}
${ }^{1}$ Department of Internal Medicine, Soonchunhyang University Bucheon Hospital, Bucheon; ${ }^{2}$ Department of Infectious Diseases, Asan Medical Center, University of Ulsan College of Medicine, Seoul; ${ }^{3}$ Center for Antimicrobial Resistance and Microbial Genetics, University of Ulsan, Seoul; ${ }^{4}$ Department of Internal Medicine, Kyung Hee University Hospital, Seoul; ${ }^{5}$ Asan Institute of Life Sciences, Asan Medical Center, University of Ulsan College of Medicine, Seoul, Korea
\end{abstract}

Received: November 3, 2016 Revised : January 23, 2017 Accepted: February 1, 2017

\section{Correspondence to}

\section{Yang Soo Kim, M.D.}

Department of Infectious Diseases, Asan Medical Center, University of Ulsan College of Medicine, 88 Olympic-ro 43-gil, Songpa-gu, Seoul 05505, Korea

Tel: $+82-2-3010-3303$

Fax: +82-2-3010-6970

E-mail:yskim@amc.seoul.kr
Background/Aims: Methicillin-resistant Staphylococcus aureus bacteremia (MRS$\mathrm{AB}$ ) is a major bloodstream infection with a high mortality rate. Identification of factors associated with early mortality in MRSAB patients would be useful for predicting prognosis and developing new therapeutic options.

Methods: A prospective cohort of MRSAB patients was examined between August 2008 and June 2011. Early and late mortality was defined as death within 2 and 28 days of blood culture, respectively. The clinical and microbiological characteristics in the early and late mortality and survival groups were compared. Risk factors associated with severe sepsis or septic shock were also investigated.

Results: A total of 385 adult MRSAB patients whose $S$. aureus isolates were available were enrolled; of these patients, 25 patients $(6.5 \%)$ and $50(13 \%)$ died early and late, respectively. Compared with both the late-mortality group and the survival group, severe sepsis or septic shock was a statistically significant independent risk factor associated with early mortality. Rapidly or ultimately fatal McCabe and Jackson classification (adjusted odds ratio [aOR], 1.94; 95\% confidence interval [CI], 1.25 to 3.02 ) and pneumonia (aOR, 2.04; $95 \%$ CI, 1.03 to 4.02 ) were independently associated with severe sepsis or septic shock. A vancomycin minimum inhibitory concentration (MIC) $\geq 1.5 \mu \mathrm{g} / \mathrm{mL}$ was associated with a reduced incidence of severe sepsis or septic shock (aOR, 0.53; 95\% CI, 0.34 to o.84).

Conclusions: Severity of illness seems to be the most important risk factor associated with early mortality in MRSAB. Although vancomycin MIC was not independently associated with early mortality, reduced vancomycin susceptibility appears to be linked to reduced disease severity.

Keywords: Methicillin-resistant Staphylococcus aureus; Bacteremia; Risk factors; Mortality

\section{INTRODUCTION}

Methicillin-resistant Staphylococcus aureus (MRSA) is a prominent cause of bloodstream infections, and the disease has a particularly high incidence and high mortal- ity rate $[1,2]$. Despite the availability of appropriate therapies, the 30-day mortality rate of patients with MRSA bloodstream infections remains at 30\% to $40 \%[3,4]$. Recent surveillance data on MRSA infections in the United States shows that $60 \%$ of in-hospital deaths occurred 
within 7 days of the initial MRSA infection [5]. One report estimates that $4.9 \%$ of patients die within 2 days after the onset of MRSA bacteremia (MRSAB) [6]. Nevertheless, most previous studies of patients with MRSAB focused on the prognostic factors associated with later mortality, such as 30-day mortality [7-13]. Only one recent study appears to have investigated the predictive factors for early mortality, defined as in-hospital death within 2 days of the onset of bacteremia, but that report did not fully investigate microbiological factors, using only an antimicrobial susceptibility test and molecular epidemiology [6]. Information on risk factors including microbiologic factors could be used to establish the prognosis for MRSAB, identify potential targets for therapeutic agents, and increase understanding of the pathogenesis of MRSA.

Using a prospective cohort of MRSAB patients, we performed a study to identify the clinical and microbiologic factors associated with early mortality. We also investigated the risk factors associated with severe sepsis or septic shock.

\section{METHODS}

\section{Study population and study design}

This prospective cohort study was conducted between August 2008 and June 2011 at the Asan Medical Center, a-2,700-bed hospital in Seoul that provides primary and tertiary care for patients from throughout Korea. By reviewing daily computerized reports on blood cultures, all patients aged $\geq 18$ years with a MRSA-positive blood culture were identified and enrolled in the study. Patients were excluded if clinical isolates of MRSA were not available for microbiologic tests, or if they had polymicrobial bacteremia or clinically insignificant MRSAB. Insignificant bacteremia was defined as satisfying all of the following conditions: isolation from only one blood culture, no clinical finding consistent with bacteremia, and no anti-staphylococcal treatment. The study consisted of two sets of analyses: early mortality versus non-early mortality, and severe sepsis or septic shock versus non-severe sepsis or septic shock (Fig. 1). Early mortality was defined as death within 2 days of blood culture, since it takes about 48 hours to react to target concentrations of vancomycin as the drug of choice
$[12,14]$. Late mortality was defined as death within 28 days of blood culture. The other patients were classified into the survival group. Severe sepsis or septic shock was defined according to International Sepsis Definitions Conference 2001 criteria [15]. The study was approved by the Institutional Review Board of Asan Medical Center (IRB No. 2008-0274).

\section{Data collection and definitions}

The following were recorded: demographic characteristics of the patients, days spent in the hospital before detection of MRSAB, underlying diseases or conditions, severity of illness, presence or absence of foreign material such as an intravenous catheter or prosthetic device, site of infection (catheter-related bloodstream infection, arteriovenous fistula infection, pneumonia, skin and soft tissue infection, bone and joint infection, urinary tract infection, and/or infective endocarditis), susceptibility to antibiotics, antibiotics used to treat MRSA (vancomycin, teicoplanin, tigecycline, and linezolid), and inhospital mortality. If the site of infection could not be determined, it was considered to be unknown. Hospitalacquired bacteremia was defined as a positive blood culture obtained from a patient who had been hospitalized for 48 hours or longer. Community-acquired bacteremia and healthcare-associated bacteremia were defined as described by Friedman et al. [16]. To estimate the severity of the illness, the sepsis grade [17] and Pitt bacteremia score [18] were obtained. To estimate the severity of comorbidities, the Charlson comorbidity score (CCS) was calculated, as previously described [19]. When blood culture was performed, disease severity was classified according to the McCabe and Jackson classification system [20]. Eradicable foci included surgically removable infections, drainable abscesses, and indwelling foreign bodies such as peripheral and central intravenous catheters [21]. Early removal of eradicable foci was defined as removal within 2 days of blood culture. Patients with non-eradicable foci and whose eradicable foci were not removed early were categorized as having non-eradicated foci. If vancomycin, teicoplanin, tigecycline, or linezolid was prescribed, the department of infectious diseases was automatically consulted and the appropriateness of the treatment was reviewed. Vancomycin dose was adjusted according to the report published by the American Society of Health-System Pharmacists [22]. 


\section{Microbiological methods}

All isolates were confirmed as MRSA by polymerase chain reaction (PCR) for the mecA gene, and tested for antimicrobial susceptibility by standard techniques according to Clinical and Laboratory Standards Institute guidelines [23]. Staphylococcal cassette chromosome (SCC) mec type MRSA was identified [24]. The presence of bacterial virulence factors, including adhesins and toxins, was examined by multiplex PCR [25,26], and multi-locus sequence typing was also performed [27]. To examine agr dysfunction, the extent of $\delta$-hemolysin production was measured by streaking each MRSA isolate next to a $\beta$-hemolysin disk (Remel, Lenexa, KS, USA) [28]. The vancomycin minimum inhibitory concentrations (MICs) were determined using the vancomycin E-test (AB Biodisk, Piscataway, NJ, USA) on Mueller-Hinton agar. Heteroresistant vancomycin-intermediate S. aureus was identified by a modified population analysis profile-area under curve ratio method [29].

\section{Statistical analysis}

Statistical analyses were performed using SPSS version 14.0 (SPSS Inc., Chicago, IL, USA). Continuous variables were compared using the Mann-Whitney U test or Student $t$ test. Categorical variables were compared using the Pearson chi-square test or Fisher exact test. Binary logistic regression was used to identify variables significantly associated with early mortality before detection of MRSAB by blood culture. Owing to the small size of the early-mortality group, the number of variables had to be minimized. Variables found to be statistically significant at the $5 \%$ level in the univariate analysis were included in the multivariate analysis. Underlying disease was introduced as a comorbidity variable only, and CCS and McCabe and Jackson classification were not included. Severe sepsis or septic shock was used to define severity of illness. Early removal of eradicable foci was also not included because of its low frequency. Anti-MRSA therapy was also excluded from the multivariate analysis because the frequency of non-treated patients was less than two in the late mortality and survival groups. Variables found to be statistically significant at the 10\% level in the univariate analysis were included in the multivariate analysis of risk factors for severe sepsis or septic shock. If no patient fit the criteria for any group, that variable was excluded from the multivariable analysis.
There was no multicollinearity among the variables included in the multivariate analysis. All significance tests were two-tailed, and a $p<0.05$ was considered significant.

\section{RESULTS}

\section{Study population}

During the study period, 577 patients with MRSAB aged $\geq 18$ years were identified. Of these patients, 192 were excluded for the following reasons: clinical isolates were not collected (117 patients), polymicrobial bacteremia was found (66 patients), or the MRSAB was clinically insignificant (nine patients). Of the 385 enrolled patients, 158 (41.0\%) died; 25 patients (6.5\%) died early and 50 patients (13\%) died within 28 days. Severe sepsis or septic shock occurred in 124 patients (32.2\%) with MRSA bacteremia. Ultimately, of 385 enrolled patients, 25 (6.5\%), 50 (13.0\%), and 310 patients (80.5\%) were assigned to the early mortality, late mortality, and survival groups (Fig. 1) Also, 124 (32.2\%) and 261 patients (67.8\%) were assigned to the severe sepsis or septic shock group and non-severe sepsis or septic shock group (Fig. 1).

\section{Risk factors associated with early mortality of pa- tients with MRSAB}

The clinical and microbiological characteristics of patients in the early mortality, late mortality, and survival groups are shown in Table 1. According to the severity index, the patients with early mortality had more severe disease than did those in the late mortality or survival groups. Early removal of eradicable foci in early mortality patients was less frequent than in the late mortality or survival groups. Of the patients treated with antibiotics, 221 (92.1\%), 14 (3.5\%), three (0.8\%), and one (0.3\%) were treated with vancomycin, teicoplanin, linezolid, and tigecycline, respectively. Some of the patients with early mortality did not receive anti-MRSA therapy within 24 hours.

ST5-SCCmecII-agrI (254 patients, 66.0\%) was the most common MRSA strain, followed by ST72-SCCmecIV-agrII (87 patients, 22.6\%) and ST239-SCCmecII-agrI (16 patients, $4.2 \%)$. The ST72-SCCmecIV-agrII strain was less often seen in the late mortality group than in the early mortality and survival groups, although this difference 
Table 1. Clinical and microbiological characteristics of patients with MRSA bacteremia according to survival

\begin{tabular}{|c|c|c|c|c|c|c|}
\hline Variable & $\begin{array}{l}\text { Early mortality } \\
\qquad(\mathrm{n}=25)\end{array}$ & $\begin{array}{l}\text { Late mortality } \\
\qquad(\mathrm{n}=50)\end{array}$ & $\begin{array}{l}\text { Survival } \\
(\mathrm{n}=310)\end{array}$ & $p$ value ${ }^{a}$ & $p$ value $^{\mathrm{b}}$ & $p$ value $^{c}$ \\
\hline \multicolumn{7}{|l|}{ Demographic } \\
\hline Age $\geq 65$ year-old & $15(60.0)$ & $27(54.0)$ & $157(50.6)$ & 0.81 & 0.41 & 0.76 \\
\hline Male & $15(60.0)$ & $34(68.0)$ & $199(64.2)$ & 0.61 & 0.67 & 0.64 \\
\hline \multicolumn{7}{|l|}{ Site of MRSA acquisition } \\
\hline Hospital-acquired & $17(68.0)$ & $42(84.0)$ & $228(73 \cdot 5)$ & 0.14 & 0.64 & 0.16 \\
\hline Healthcare-associated & $7(28.0)$ & $7(14.0)$ & $68(21.9)$ & 0.21 & 0.46 & 0.26 \\
\hline Community-acquired & $1(4.0)$ & $1(2.0)$ & $14(4 \cdot 5)$ & 1.00 & 1.00 & 0.70 \\
\hline \multicolumn{7}{|l|}{ Length of hospital stay before MRSA detection } \\
\hline$<72$ hours & $9(36.0)$ & $9(18.0)$ & $92(29.7)$ & 0.10 & 0.50 & 0.09 \\
\hline $3-7$ days & $2(8.0)$ & $4(8.0)$ & $27(8.7)$ & 1.00 & 1.00 & 1.00 \\
\hline $8-28$ days & $10(40.0)$ & $25(50.0)$ & $122(39.4)$ & 0.47 & 1.00 & 0.17 \\
\hline$>28$ days & $4(16.0)$ & $12(24 \cdot 0)$ & $69(22.3)$ & 0.56 & 0.62 & 0.86 \\
\hline \multicolumn{7}{|l|}{ Underlying disease } \\
\hline Solid tumor & $14(56.0)$ & $25(50.0)$ & $115(37 \cdot 1)$ & 0.81 & 0.09 & 0.09 \\
\hline Hematologic malignancy & $1(4 \cdot 0)$ & $7(14.0)$ & $19(6.1)$ & 0.26 & 1.00 & 0.07 \\
\hline ESRD & ० & $3(6.0)$ & $39(12.6)$ & 0.55 & 0.10 & 0.24 \\
\hline Liver cirrhosis & $8(32.0)$ & $8(16.0)$ & $34(11.0)$ & 0.14 & 0.01 & 0.34 \\
\hline Cardiovascular disease & $5(20.0)$ & $10(20.0)$ & $55(17 \cdot 7)$ & 1.00 & 0.79 & 0.69 \\
\hline \multicolumn{7}{|l|}{ Severity of comorbidity } \\
\hline Charlson comorbidity score $\geq 5$ & $12(48.0)$ & $22(44.0)$ & $68(21.9)$ & 0.81 & 0.01 & $<0.001$ \\
\hline \multicolumn{7}{|l|}{ McCabe and Jackson classification } \\
\hline Rapidly or ultimately fatal & $20(80.0)$ & $35(70.0)$ & $97(31.3)$ & 0.42 & $<0.001$ & $<0.001$ \\
\hline \multicolumn{7}{|l|}{ Site of infection } \\
\hline Catheter-related blood stream infection & $9(36.0)$ & $24(48.0)$ & $141(45 \cdot 5)$ & 0.46 & 0.41 & 0.76 \\
\hline Arteriovenous fistula infection & o & O & $6(1.9)$ & NA & 1.00 & 1.00 \\
\hline Pneumonia & $5(20.0)$ & $5(10.0)$ & $30(9,7)$ & 0.29 & 0.16 & 1.00 \\
\hline Skin and soft tissue infection & o & $3(6.0)$ & $12(3.9)$ & 0.55 & 0.61 & 0.45 \\
\hline Surgical site infection & $2(8.0)$ & $2(4 \cdot 0)$ & $32(10.3)$ & 0.60 & 1.00 & 0.20 \\
\hline Bone and joint infection & 0 & $1(2.0)$ & $18(5.8)$ & 1.00 & 0.38 & 0.49 \\
\hline Urinary tract infection & 0 & 0 & $3(1.0)$ & NA & 1.00 & 1.00 \\
\hline Infective endocarditis & $1(4.0)$ & $3(6.0)$ & $5(1.6)$ & 1.00 & 0.37 & 0.09 \\
\hline Unknown & $8(32.0)$ & $24(48.0)$ & $141(45 \cdot 5)$ & 0.46 & 0.41 & 0.05 \\
\hline \multicolumn{7}{|l|}{ Severity of illness } \\
\hline Severe sepsis or septic shock & $20(80.0)$ & $27(54.0)$ & $77(24.8)$ & 0.04 & $<0.001$ & $<0.001$ \\
\hline Pitt bacteremia score $\geq 3$ & $17(68.0)$ & $27(54.0)$ & $73(23 \cdot 5)$ & 0.004 & $<0.001$ & $<0.001$ \\
\hline Eradicable foci & $10(43.6)$ & $28(59.6)$ & $194(64.9)$ & 0.31 & 0.046 & 0.51 \\
\hline Early removal & $2(16.7)$ & $24(75 \cdot 0)$ & $175(78.1)$ & 0.001 & $<0.001$ & 0.66 \\
\hline \multicolumn{7}{|l|}{ Anti-MRSA therapy } \\
\hline On day o & $12(48.0)$ & $24(48.0)$ & $79(25 \cdot 5)$ & 1.00 & 0.02 & 0.002 \\
\hline On day o or 1 & $16(64.0)$ & $50(100)$ & $308(99.4)$ & $<0.001$ & $<0.001$ & 1.00 \\
\hline Microbiologic factors & & & & & & \\
\hline
\end{tabular}


Table 1. Continued

\begin{tabular}{|c|c|c|c|c|c|c|}
\hline Variable & $\begin{array}{l}\text { Early mortality } \\
\qquad(\mathrm{n}=25)\end{array}$ & $\begin{array}{l}\text { Late mortality } \\
\qquad(\mathrm{n}=50)\end{array}$ & $\begin{array}{l}\text { Survival } \\
(\mathrm{n}=310)\end{array}$ & $p$ value $^{\mathrm{a}}$ & $p$ value $^{b}$ & $p$ value $^{c}$ \\
\hline Vancomycin MIC by E-test $\geq 1.5 \mu \mathrm{g} / \mathrm{mL}$ & $13(52.0)$ & $35(70.0)$ & $216(69.7)$ & 0.14 & 0.08 & 1.00 \\
\hline hVISA phenotype & $5(20.0)$ & $14(28.0)$ & $94(30.3)$ & 0.58 & 0.19 & 0.87 \\
\hline ST72-SCCmecIV-agrI strain & $8(32.0)$ & $4(8.0)$ & $74(23.9)$ & 0.02 & 0.34 & 0.009 \\
\hline agr dysfunction & $18(72.0)$ & $37(74 \cdot 0)$ & $211(68.1)$ & 1.00 & 1.00 & 0.30 \\
\hline \multicolumn{7}{|l|}{ Virulence genes } \\
\hline$s d r C$ & $17(68.0)$ & $43(86.0)$ & $261(84.2)$ & 0.12 & 0.05 & 0.84 \\
\hline $\sec$ & $16(64.0)$ & $37(74.0)$ & $204(65.8)$ & 0.43 & 0.83 & 0.33 \\
\hline sel & $16(64.0)$ & $44(88.0)$ & $232(74.8)$ & 0.03 & 0.24 & 0.047 \\
\hline TSST-1 & $14(56.0)$ & $40(80.0)$ & $207(66.8)$ & 0.05 & 0.28 & 0.07 \\
\hline
\end{tabular}

Values are presented as number (\%).

MRSA, methicillin-resistant Staphylococcus aureus; ESRD, end-stage renal disease; NA, not applicable; MIC, microbacterial inhibitory concentration; hVISA, heterogeneous vancomycin-intermediate Staphylococcus aureus; ST, sequence type; SCC, staphylococcal cassette chromosome; TSST-1, toxic shock syndrome toxin 1.

${ }^{a} p$ values for comparison between the early and late mortality groups.

${ }^{\mathrm{b}} \mathrm{p}$ values for comparison between the early mortality and survival groups.

${ }^{c} p$ values for comparison between the late mortality and survival groups.

was not statistically significant in the multivariate analysis. The distribution of vancomycin MICs was as follows: in 121 cases $(31.4 \%)$ it was $\leq 1.0 \mu \mathrm{g} / \mathrm{mL}$, in $177(45.9 \%)$ it was $\leq 1.5 \mu \mathrm{g} / \mathrm{mL}$, in $82(21.3 \%)$ it was $\leq 2.0 \mu \mathrm{g} / \mathrm{mL}$, and in $5(1.8 \%)$ it was $>2 \mu \mathrm{g} / \mathrm{mL}$. The following virulent genes were not found in any isolates: seb, sed, see, seh, sej, eta, etb, $l u k M, h l g$, and edin. Regardless of survival, the following genetic factors were very rare: Panton-Valentine leukocidin, map/eap, sea, sek, sep, and seq. Virulent genes such as fnbA, clfA, clfB, can, and icaA were found in all isolates, and frbB, bbp, ebps, sdrD, sdrE, seg, sei, sem, sen, seo, lukDE, lukE, hla, hld, and hlg2 were found in most isolates. The frequencies of virulent genes such as $s d r C$, sec, sel, and TSST-1 are given in Table 1. Among the virulent genes, sel was less frequent in patients with early mortality than in those with late mortality.

To compare the clinical and microbiologic factors seen in patients with early mortality versus those with late mortality, occurrence of severe sepsis or septic shock, presence of the ST72-SCCmecIV-agrI strain, and sel as a virulent gene were included in the multivariate analysis (Table 2). Compared with patients with late mortality, severe sepsis or septic shock (adjusted odds ratio [aOR], 4.49; $95 \%$ confidence interval [CI], 1.28 to 15.75 ) was the only statistically significant independent risk factor in patients with early mortality. To compare the clinical and microbiologic factors of patients with early mortality and those who survived, the following variables were included in the multivariate analysis: liver cirrhosis, severe sepsis or septic shock, and anti-MRSA therapy on day of blood culture (Table 2). Compared with patients who survived, liver cirrhosis (aOR, 3.79; 95\% CI, 1.38 to 23.86) and severe sepsis or septic shock (aOR, 10.98; $95 \%$ CI, 3.82 to 31.52 ) were statistically significant independent risk factors in patients with early mortality. When rapidly or ultimately fatal McCabe and Jackson classification was added to the multivariate analysis and severe sepsis or septic shock was replaced with Pitt bacteremia score $\geq 3$, both were also statistically significant (data not shown). Compared with patients who survived, rapidly or ultimately fatal McCabe and Jackson classification (aOR, 4.61; 95\% CI, 2.37 to 9.00) and severe sepsis or septic shock (aOR, 2.95; 95\% CI, 1.54 to 5.63) were statistically significant independent risk factors in patients with late mortality.

\section{Risk factors associated with severe sepsis or septic shock}

The clinical and microbiologic characteristics of patients in the severe sepsis or septic shock and non-severe sepsis or septic shock groups are shown in Table 3. In the multivariate analysis, rapidly or ultimately fatal 
Table 2. Risk factors associated with early mortality in patients with MRSA bacteremia according to survival

\begin{tabular}{|c|c|c|c|c|c|c|}
\hline \multirow[b]{2}{*}{ Variable } & \multicolumn{2}{|c|}{ Early mortality vs. late mortality } & \multicolumn{2}{|c|}{ Early mortality vs. survival } & \multicolumn{2}{|c|}{ Late mortality vs. survival } \\
\hline & $\begin{array}{c}\text { Univariate } \\
\text { analysis, OR } \\
(95 \% \mathrm{CI})\end{array}$ & $\begin{array}{l}\text { Multivariate } \\
\text { analysis, aOR } \\
(95 \% \mathrm{CI})\end{array}$ & $\begin{array}{c}\text { Univariate } \\
\text { analysis, OR } \\
(95 \% \mathrm{CI})\end{array}$ & $\begin{array}{l}\text { Multivariate } \\
\text { analysis, aOR } \\
(95 \% \mathrm{CI})\end{array}$ & $\begin{array}{c}\text { Univariate } \\
\text { analysis, OR } \\
(95 \% \mathrm{CI})\end{array}$ & $\begin{array}{l}\text { Multivariate } \\
\text { analysis, aOR } \\
(95 \% \mathrm{CI})\end{array}$ \\
\hline \multicolumn{7}{|l|}{ Comorbidity } \\
\hline Liver cirrhosis & - & - & $2.92(1.52-5.61)$ & $3.79(1.38-10.37)^{\mathrm{a}}$ & - & - \\
\hline \multicolumn{7}{|l|}{$\begin{array}{l}\text { McCabe and Jackson } \\
\text { classification }\end{array}$} \\
\hline $\begin{array}{l}\text { Rapidly or ultimately } \\
\text { fatal }\end{array}$ & - & - & - & - & $2.24(1.75-2.86)$ & $4.61(2.37-9.00)^{a}$ \\
\hline \multicolumn{7}{|l|}{ Severity of illness } \\
\hline $\begin{array}{l}\text { Severe sepsis or septic } \\
\text { shock }\end{array}$ & $1.48(1.07-2.05)$ & $4.49(1.28-15.75)^{\mathrm{a}}$ & $3.22(2.45-4.24)$ & $10.98(3.82-31.52)^{\mathrm{a}}$ & $2.17(1.58-3.00)$ & $2.95(1.54-5.63)^{\mathrm{a}}$ \\
\hline \multicolumn{7}{|l|}{ Treatment } \\
\hline $\begin{array}{l}\text { Anti-MRSA therapy } \\
\text { on Do }\end{array}$ & - & - & $1.88(1.20-2.96)$ & $1.41(0.57-3.50)$ & - & - \\
\hline \multicolumn{7}{|l|}{ Microbiologic factors } \\
\hline $\begin{array}{l}\text { ST72-SCCmecIV-agrI } \\
\text { strain }\end{array}$ & $3.20(1.17-8.78)$ & $3.14(0.41-23.86)$ & - & - & $1.18\left(1.05^{-1.32)}\right.$ & $1.75(0.20-1.69)$ \\
\hline sel & $0.73(0.53-0.99)$ & $0.43(0.07-2.77)$ & - & - & $0.48(0.22-1.04)$ & $0.58(0.20-1.69)$ \\
\hline
\end{tabular}

OR, odds ratio; CI, confidence interval; aOR, adjusted odds ratio; MRSA, methicillin-resistant Staphylococcus aureus; ST, sequence type; SCC, staphylococcal cassette chromosome.

${ }^{\mathrm{a}} \mathrm{p}<0.05$.

McCabe and Jackson classification as severity of comorbidity (aOR, 1.94; 95\% CI, 1.25 to 3.02) and pneumonia as site of infection (aOR, 2.04; 95\% CI, 1.03 to 4.02 ) were significantly associated with severe sepsis or septic shock. Fewer patients with isolates with vancomycin MICs $\geq 1.5$ $\mu \mathrm{g} / \mathrm{mL}$ experienced severe sepsis or septic shock (aOR, 0.53; $95 \%$ CI, 0.33 to 0.84 ).

\section{DISCUSSION}

The main aim of the current study was to identify clinical factors associated with early death in patients with MRSAB. Identifying key factors could help to identify those patients who require urgent and aggressive management. Liver cirrhosis and severe sepsis or septic shock were independent risk factors associated with early mortality. The secondary aim of our study was to investigate the microbiological factors associated with early mortality. Paradoxically, low vancomycin MIC was associated with severe sepsis or septic shock, although vancomycin susceptibility was not associated with early mortality. To our knowledge, this is the first study to extensively investigate the microbiological factors associated with early mortality in MRSAB patients.

Indices of the severity of comorbidities and illness are known to be associated with death of patients with $S$. aureus bacteremia (SAB) [11-13,30,31], and most previous studies investigated the factors associated with late mortality, such as 30-day mortality [11-13,30,31]. The exception is a recent study by Gasch et al. [6], which looked at early mortality, defined as death within 2 days of the onset of MRSAB, as in the current study. According to Gasch and colleagues [6], rapidly fatal McCabe and Jackson classification (aOR, 3.67; 95\% CI, 1.32 to 10.24) and Pitt bacteremia score > 3 (aOR, 4.52; 95\% CI, 1.72 to 9.24) were independent factors associated with early mortality. Our current findings also suggest that the severities of comorbidities and illness are important prognostic factors that influence early death as well as late death in patients with MRSAB.

Underlying liver cirrhosis was significantly associated 
Table 3. Risk factors associated with severe sepsis or septic shock in patients with MRSA bacteremia

\begin{tabular}{|c|c|c|c|c|c|}
\hline Variable & $\begin{array}{c}\text { Severe sepsis or } \\
\text { septic shock }(n=124)\end{array}$ & $\begin{array}{l}\text { Non-severe sepsis or } \\
\text { septic shock }(n=261)\end{array}$ & $p$ value & $\begin{array}{l}\text { Univariate analy- } \\
\text { sis, OR (95\% CI) }\end{array}$ & $\begin{array}{l}\text { Multivariate analy- } \\
\text { sis, aOR (95\% CI) }\end{array}$ \\
\hline \multicolumn{6}{|l|}{ Demographic } \\
\hline Age $\geq 65$ year-old & $68(54.8)$ & $131(50.2)$ & 0.45 & $1.21(0.79-1.85)$ & - \\
\hline Male sex & $83(66.9)$ & $165(63.2)$ & 0.50 & $1.18(0.75-1.85)$ & - \\
\hline \multicolumn{6}{|l|}{ Site of MRSA acquisition } \\
\hline Hospital-acquired & $92(74.2)$ & $195(74.7)$ & 0.90 & $0.97(0.60-1.59)$ & - \\
\hline Healthcare-associated & $27(21.8)$ & $55(21.1)$ & 0.89 & $1.04(0.62-1.75)$ & - \\
\hline Community-acquired & $5(4.0)$ & $11(4.2)$ & 1.00 & $0.96(0.32-2.81)$ & - \\
\hline \multicolumn{6}{|c|}{ Length of hospital stay before MRSA detection } \\
\hline$<72$ hours & $37(29.8)$ & $73(28.0)$ & 0.72 & $1.10(0.68-1.75)$ & - \\
\hline $3-7$ days & $10(8.1)$ & $23(8.0)$ & 1.00 & $0.91(0.42-1.97)$ & - \\
\hline $8-28$ days & $47(37.9)$ & $110(42.1)$ & 0.44 & $0.84(0.54-1.30)$ & - \\
\hline$>28$ days & $30(24.2)$ & $55(21.1)$ & 0.51 & $1.20(0.72-1.99)$ & - \\
\hline \multicolumn{6}{|l|}{ Underlying disease } \\
\hline Solid tumor & $48(38.7)$ & $106(40.6)$ & 0.74 & $0.92(0.60-1.43)$ & - \\
\hline Hematologic malignancy & $11(8.9)$ & $16(6.1)$ & 0.39 & $1.49(0.67-9.32)$ & - \\
\hline ESRD & $14(11.3)$ & $28(10.7)$ & 0.86 & $1.06(0.54-2.09)$ & - \\
\hline Liver cirrhosis & $17(13.7)$ & $33(12.6)$ & 0.75 & $1.10(0.59-2.06)$ & - \\
\hline Cardiovascular disease & $26(21.0)$ & $44(16.9)$ & 0.33 & $1.31(0.76-2.25)$ & - \\
\hline \multicolumn{6}{|l|}{ Severity of comorbidity } \\
\hline Charlson comorbidity score $\geq 5$ & $36(29.0)$ & $66(25 \cdot 3)$ & 0.46 & $1.21(0.75-1.95)$ & - \\
\hline \multicolumn{6}{|l|}{ McCabe and Jackson classification } \\
\hline Rapidly or ultimately fatal & $63(50.8)$ & $89(34.1)$ & 0.003 & $2.00(1.29-3.08)$ & $1.94(1.25-3.02)$ \\
\hline \multicolumn{6}{|l|}{ Site of infection } \\
\hline $\begin{array}{l}\text { Catheter-related blood stream } \\
\text { infection }\end{array}$ & $60(48.4)$ & $114(43.7)$ & 0.44 & $1.21(0.79-1.86)$ & - \\
\hline Arteriovenous fistula infection & $1(0.8)$ & $5(1.9)$ & 0.67 & $0.42(0.05-3.60)$ & - \\
\hline Pneumonia & $19(15 \cdot 3)$ & $21(8.0)$ & 0.03 & $2.07(1.07-4.01)$ & $2.04(1.03-4.02)$ \\
\hline Skin and soft tissue infection & o & $15(5 \cdot 7)$ & 0.004 & NA & - \\
\hline Surgical site infection & $9(7 \cdot 3)$ & $27(10.3)$ & 0.45 & $0.68(0.31-1.49)$ & - \\
\hline Bone and joint infection & $5(4.0)$ & $14(5 \cdot 4)$ & 0.80 & $0.74(0.26-2.11)$ & - \\
\hline Urinary tract infection & $1(0.8)$ & $2(0.8)$ & 1.00 & $1.05(0.10-11.72)$ & - \\
\hline Infective endocarditis & $3(2.4)$ & $6(2.3)$ & 1.00 & $1.05(0.26-4.29)$ & - \\
\hline Unknown & $20(16.1)$ & $29(11.1)$ & 0.19 & $1.54(0.83-2.85)$ & - \\
\hline Early mortality & $20(16.1)$ & $5(1.9)$ & $<0.001$ & $9.85(3.60-26.93)$ & - \\
\hline \multicolumn{6}{|l|}{ Microbiologic factors } \\
\hline $\begin{array}{l}\text { Vancomycin MIC by E-test } \geq \\
1.5 \mu \mathrm{g} / \mathrm{mL}\end{array}$ & $73(58.9)$ & $191(73.2)$ & 0.01 & $0.53(0.33-0.82)$ & $0.53(0.34-0.84)$ \\
\hline hVISA phenotype & $37(32.5)$ & $76(32.6)$ & 1.00 & $0.99(0.62-1.60)$ & - \\
\hline \multicolumn{6}{|l|}{ Strain } \\
\hline ST5-SCCmecII-agrII strain & $80(71.0)$ & $165(63.2)$ & 0.17 & $1.42(0.90-2.26)$ & - \\
\hline ST72-SCCmecIV-agrI strain & $23(18.5)$ & $63(24.1)$ & 0.24 & $0.72(0.42-1.22)$ & - \\
\hline agr dysfunction & $89(74.2)$ & $177(71.1)$ & 0.62 & $1.17(0.71-1.91)$ & - \\
\hline
\end{tabular}

Values are presented as number (\%).

MRSA, methicillin-resistant Staphylococcus aureus; OR, odds ratio; CI, confidence interval; aOR, adjusted odds ratio; ESRD, endstage renal disease; NA, not applicable; MIC, microbacterial inhibitory concentration; hVISA, heterogeneous vancomycin-intermediate Staphylococcus aureus; ST, sequence type; SCC, staphylococcal cassette chromosome. 


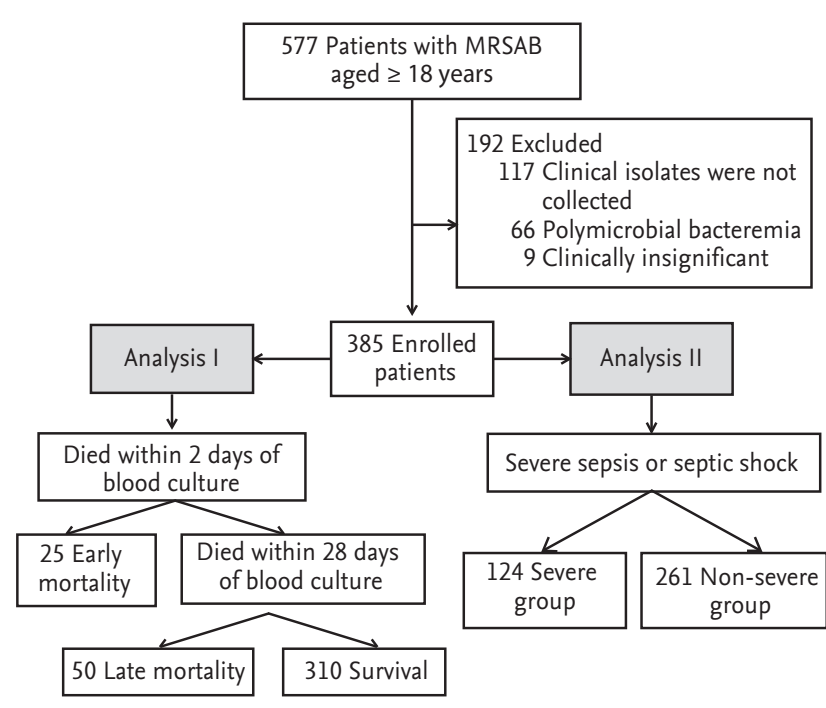

Figure 1. Algorithm of enrollment and analysis. MRSAB, methicillin-resistant Staphylococcus aureus bacteremia.

with early mortality in the present analysis. A previously published report identified liver cirrhosis an independent predictor of SAB-related mortality [21]. The association can be explained by weakened host defense in patients with liver cirrhosis, including impaired functioning of leukocytes [32] and Kupffer cells [33]. This association implies that patients with liver cirrhosis may require more careful and intensive management beginning in the early phase of infection.

Another important issue is whether treatment with empirical antibiotics can prevent early death from MRSA. If this is the case, such antibiotics should be administered without hesitation to patients with any risk factors associated with MRSA infection immediately after blood culture. Gasch et al. [6] reported that inappropriate antibiotic usage within 48 hours of MRSAB onset was independently associated with early mortality (aOR, 3.59; $95 \%$ CI, 1.63 to 7.89). Although we could not examine this issue in the multivariate analysis, our study also showed that patients with early mortality did not receive appropriate antibiotics on days o and 1 compared to patients with late morality and survivors. However, these results may have been caused by a survival bias.

One of the most interesting findings of the present study concerns the paradoxical association of reduced vancomycin susceptibility with severe sepsis or septic shock. This paradoxical relationship is in disagreement with a previous study on early SAB mortality [6]. Gasch et al. [6] reported a trend towards a higher proportion of isolates with vancomycin MICs $\geq 1.5 \mu \mathrm{g} / \mathrm{L}$ in patients with early mortality (early mortality 8.0\% [4/49] vs. non-early mortality $3.0 \%[14 / 530], p=0.05)$ in their univariate analysis. In their work, inappropriate antibiotic usage within 48 hours after MRSAB onset was independently associated with early mortality. The association of high vancomycin MIC with early mortality would imply that use of higher doses of appropriate empiric glycopeptide therapies are important for patient survival. In our study, in contrast, low vancomycin susceptibility was associated with a lower risk of severe sepsis or septic shock. In a prospective cohort study of MRSAB. Soriano et al. [9] also showed that episodes caused by strains with a vancomycin MIC of $2 \mathrm{mg} / \mathrm{L}$ were independently associated with a lower risk of shock (OR, 0.33; 95\% CI, 1.68 to 24.3), as seen in this study. Using the non-mammalian model system Galleria mellonella (the wax moth), Peleg et al. [34] showed that killing was significantly attenuated after infection with a vancomycin-non-susceptible $S$. aureus strain in contrast with an isogenic, vancomycin-susceptible progenitor strain. This would mean that higher doses or loading doses of glycopeptides may have only a limited role in reducing the severity of MRSA bacteremia.

These discordant results on the relationship of vancomycin MIC may be attributable to the fact that severe sepsis or septic shock has different properties from early mortality as an outcome indicator. Patients with early mortality were often classified as rapidly or fatal McCabe classification as an indicator of comorbidity, and it can be assumed that these patients were more likely to be exposed to antibiotics prior to MRSA infection. It is already known that exposure to glycopeptides increases MIC as the cell wall becomes thicker. Cui et al. [35] found that cells with thin cell walls displayed reduced growth in the presence of vancomycin compared to cells with thick cell walls. Therefore, the positive correlation between early mortality and vancomycin MIC values seen in a previous study can be interpreted as the result of a comorbidity rather than a risk factor affecting early mortality. On the other hand, severe sepsis or severe septic shock as an indicator of outcome is the result of host immune activation. It can be inferred that vancomycin MIC is inversely correlated because a strain with 
a high vancomycin MIC induces less host immune activation, as it is a 'fitness cost.' A thickened cell wall can prevent teichoic acids and lipoteichoic acids from activating the immune system and consequently hinder the development of septic shock [9]. MRSA strains with high vancomycin MICs also tend to have slow growth rates [36]. Further well-designed studies that control bias due to non-microbiological factors should be undertaken to better understand the impact of reduced vancomycin susceptibility on early prognosis in MRSAB.

The $\mathrm{ST}_{72}$-SCCmecIV-agrI strain was more prevalent in the early mortality group than in the late mortality group in the univariate analysis. This is in agreement with the result of a previous study conducted by our colleagues in the same population, which showed that the community-associated MRSA strain ST72-SCCmecIV was independently associated with low crude mortality, compared to the ST5-SCCmecII strain (aOR, 0.26; 95\% CI, 0.13 to 0.54 ) [37]. Also, sel, one of the staphylococcal superantigen genes that cause immune system dysregulation, was less frequent in the early mortality group. The patterns of strain type and virulent gene were similar in the early mortality group and survival groups, but different in the late mortality group. These unexpected results may be because host immune activation has a stronger influence on early mortality, and may offset the influence of microbiological factors as prognostic factors.

This study had several limitations. First, some variables that affect the outcomes of MRSAB patients may have been omitted from the analysis. Second, early death in some patients might not have been caused by MRSAB, but rather by alternative causes such as terminal cancer or impediments to care such as refusal of intensive care. A third limitation is the narrow range of ST types in our sample. Studies from different regions or countries that include various ST types of MRSA strains should be performed. Finally, the small size of the early mortality group is likely to have been insufficient to draw firm conclusions about whether any microbiological factors affect early mortality.

In conclusion, comorbidities such as liver cirrhosis and severity of illness such as severe sepsis or septic shock are important risk factors for early mortality in MRSAB, just as in late mortality. In the current situation, it seems that early intervention can play only a limited role in improving early prognosis in MRSA, and this conclusion emphasizes the importance of preventing MRSAB. The paradoxical relationship between vancomycin susceptibility and severe sepsis or septic shock suggests that these isolates may lose their virulence when acquiring vancomycin resistance. Further studies are required to explain the associations of these clinical and microbiological factors with early prognosis in patients with MRSAB.

\section{KEY MESSAGE}

1. About $7 \%$ of patients with methicillin-resistant Staphylococcus aureus bacteremia died within 2 days of blood culture.

2. Liver cirrhosis and severe sepsis or septic shock were independent clinical factors associated with early mortality.

3. Reduced vancomycin susceptibility appears to be a linked to reduced disease severity.

\section{Conflict of interest}

No potential conflict of interest relevant to this article was reported.

\section{Acknowledgments}

This work was supported by grants from the Korean Health Technology R\&D Project, Ministry of Health and Welfare, Republic of Korea (HI15 2918 ).

\section{REFERENCES}

1. Weinstein MP, Towns ML, Quartey SM, et al. The clinical significance of positive blood cultures in the 1990s: a prospective comprehensive evaluation of the microbiology, epidemiology, and outcome of bacteremia and fungemia in adults. Clin Infect Dis 1997;24:584-602.

2. Laupland KB, Ross T, Gregson DB. Staphylococcus aureus bloodstream infections: risk factors, outcomes, and the influence of methicillin resistance in Calgary, Canada, 2000-2006. J Infect Dis 2008;198:336-343.

3. Gasch O, Ayats J, Angeles Dominguez M, et al. Epidemiology of methicillin-resistant Staphylococcus aureus (MRSA) bloodstream infection: secular trends over 19 years at a university hospital. Medicine (Baltimore) 2011; 
90:319-327.

4. Paul M, Kariv G, Goldberg E, et al. Importance of appropriate empirical antibiotic therapy for methicillin-resistant Staphylococcus aureus bacteraemia. J Antimicrob Chemother 2010;65:2658-2665.

5. Dantes R, Mu Y, Belflower R, et al. National burden of invasive methicillin-resistant Staphylococcus aureus infections, United States, 2011. JAMA Intern Med 2013;173:19701978.

6. Gasch O, Camoez M, Dominguez MA, et al. Predictive factors for early mortality among patients with methicillin-resistant Staphylococcus aureus bacteraemia. J Antimicrob Chemother 2013;68:1423-1430.

7. Wang JT, Wu HS, Weng CM, Hsu LY, Wang FD. Prognosis of patients with methicillin-resistant Staphylococcus aureus bloodstream infection treated with teicoplanin: a retrospective cohort study investigating effect of teicoplanin minimum inhibitory concentrations. BMC Infect Dis 2013;13:182.

8. Moore CL, Lu M, Cheema F, et al. Prediction of failure in vancomycin-treated methicillin-resistant Staphylococcus aureus bloodstream infection: a clinically useful risk stratification tool. Antimicrob Agents Chemother 2011;55:4581-4588.

9. Soriano A, Marco F, Martinez JA, et al. Influence of vancomycin minimum inhibitory concentration on the treatment of methicillin-resistant Staphylococcus aureus bacteremia. Clin Infect Dis 2008;46:193-200.

10. Walraven CJ, North MS, Marr-Lyon L, Deming P, Sakoulas G, Mercier RC. Site of infection rather than vancomycin MIC predicts vancomycin treatment failure in methicillin-resistant Staphylococcus aureus bacteraemia. J Antimicrob Chemother 2011;66:2386-2392.

11. Gasch O, Camoez M, Dominguez MA, et al. Predictive factors for mortality in patients with methicillin-resistant Staphylococcus aureus bloodstream infection: impact on outcome of host, microorganism and therapy. Clin Microbiol Infect 2013;19:1049-1057.

12. Kim J, Joo EJ, Ha YE, et al. Impact of a computerized alert system for bacteremia notification on the appropriate antibiotic treatment of Staphylococcus aureus bloodstream infections. Eur J Clin Microbiol Infect Dis 2013;32:937-945.

13. Wi YM, Kim JM, Joo EJ, et al. High vancomycin minimum inhibitory concentration is a predictor of mortality in meticillin-resistant Staphylococcus aureus bacteraemia. Int J Antimicrob Agents 2012;40:108-113.
14. Wysocki M, Delatour F, Faurisson F, et al. Continuous versus intermittent infusion of vancomycin in severe Staphylococcal infections: prospective multicenter randomized study. Antimicrob Agents Chemother 2001;45:2460-2467.

15. Levy MM, Fink MP, Marshall JC, et al. 2001 SCCM/ESICM/ACCP/ATS/SIS international sepsis definitions conference. Intensive Care Med 2003;29:530-538.

16. Friedman ND, Kaye KS, Stout JE, et al. Health care: associated bloodstream infections in adults. A reason to change the accepted definition of community-acquired infections. Ann Intern Med 2002;137:791-797.

17. Bone RC, Balk RA, Cerra FB, et al. Definitions for sepsis and organ failure and guidelines for the use of innovative therapies in sepsis. The ACCP/SCCM Consensus Conference Committee. American College of Chest Physicians/ Society of Critical Care Medicine. Chest 1992;101:16441655 .

18. Paterson DL, Ko WC, Von Gottberg A, et al. International prospective study of Klebsiella pneumoniae bacteremia: implications of extended-spectrum beta-lactamase production in nosocomial Infections. Ann Intern Med 2004;140:26-32.

19. Charlson ME, Pompei P, Ales KL, MacKenzie CR. A new method of classifying prognostic comorbidity in longitudinal studies: development and validation. J Chronic Dis 1987;40:373-383.

20. McCabe WR, Jackson GG. Gram-negative bacteremia. 1. Etiology and ecology. Arch Intern Med 1962;110:847-855.

21. Kim SH, Park WB, Lee KD, et al. Outcome of Staphylococcus aureus bacteremia in patients with eradicable foci versus noneradicable foci. Clin Infect Dis 2003;37:794-799.

22. Rybak M, Lomaestro B, Rotschafer JC, et al. Therapeutic monitoring of vancomycin in adult patients: a consensus review of the American Society of Health-System Pharmacists, the Infectious Diseases Society of America, and the Society of Infectious Diseases Pharmacists. Am J Health Syst Pharm 2009;66:82-98.

23. Clinical and Laboratory Standards Institute. Performance Standards for Antimicrobial Susceptibility Testing: Eighteenth Informational Supplement. Wayne: Clinical and Laboratory Standards Institute, 2008.

24. Oliveira DC, de Lencastre H. Multiplex PCR strategy for rapid identification of structural types and variants of the mec element in methicillin-resistant Staphylococcus aureus. Antimicrob Agents Chemother 2002;46:2155-2161. 25. Diep BA, Carleton HA, Chang RF, Sensabaugh GF, Per- 
dreau-Remington F. Roles of 34 virulence genes in the evolution of hospital- and community-associated strains of methicillin-resistant Staphylococcus aureus. J Infect Dis 2006;193:1495-1503.

26. Peacock SJ, Moore CE, Justice A, et al. Virulent combinations of adhesin and toxin genes in natural populations of Staphylococcus aureus. Infect Immun 2002;70:49874996.

27. Enright MC, Day NP, Davies CE, Peacock SJ, Spratt BG. Multilocus sequence typing for characterization of methicillin-resistant and methicillin-susceptible clones of Staphylococcus aureus. J Clin Microbiol 2000;38:10081015 .

28. Schweizer ML, Furuno JP, Sakoulas G, et al. Increased mortality with accessory gene regulator (agr) dysfunction in Staphylococcus aureus among bacteremic patients. Antimicrob Agents Chemother 2011;55:1082-1087.

29. Wootton M, Howe RA, Hillman R, Walsh TR, Bennett PM, MacGowan AP. A modified population analysis profile (PAP) method to detect hetero-resistance to vancomycin in Staphylococcus aureus in a UK hospital. J Antimicrob Chemother 2001;47:399-403.

30. Park HJ, Lee YM, Bang KM, et al. Clinical significance of Staphylococcus aureus bacteremia in patients with liver cirrhosis. Eur J Clin Microbiol Infect Dis 2012;31:33093316.
31. Rieg S, Peyerl-Hoffmann G, de With K, et al. Mortality of S. aureus bacteremia and infectious diseases specialist consultation: a study of 521 patients in Germany. J Infect 2009;59:232-239.

32. Claria J, Titos E, Jimenez W, et al. Altered biosynthesis of leukotrienes and lipoxins and host defense disorders in patients with cirrhosis and ascites. Gastroenterology 1998;115:147-156.

33. Bilzer M, Roggel F, Gerbes AL. Role of Kupffer cells in host defense and liver disease. Liver Int 2006;26:1175-1186.

34. Peleg AY, Monga D, Pillai S, Mylonakis E, Moellering RC Jr, Eliopoulos GM. Reduced susceptibility to vancomycin influences pathogenicity in Staphylococcus aureus infection. J Infect Dis 2009;199:532-536.

35. Cui L, Iwamoto A, Lian JQ, et al. Novel mechanism of antibiotic resistance originating in vancomycin-intermediate Staphylococcus aureus. Antimicrob Agents Chemother 2006;50:428-438.

36. Pfeltz RF, Singh VK, Schmidt JL, et al. Characterization of passage-selected vancomycin-resistant Staphylococcus aureus strains of diverse parental backgrounds. Antimicrob Agents Chemother 2000;44:294-303.

37. Park KH, Chong YP, Kim SH, et al. Community-associated MRSA strain ST72-SCCmecIV causing bloodstream infections: clinical outcomes and bacterial virulence factors. J Antimicrob Chemother 2015;70:1185-1192. 